\title{
Why Does Academic Medicine Allow Ghostwriting? A Prescription for Reform
}

\author{
Jonathan Leo • Jeffrey R. Lacasse • Andrea N. Cimino
}

Published online: 21 July 2011

(C) Springer Science + Business Media, LLC 2011

ghost writer (n., orig. U.S.): a hack writer who does work for which another person takes the credit - Oxford English Dictionary

A book, paper, or speech that involves an author who is not given credit is considered ghostwritten, at least according to most dictionary definitions. This straightforward and seemingly commonsense definition has yet to be accepted within academic medicine. Over the past 15 years, the academic medical community has quietly tolerated the presence of ghostwriters in the medical literature, a practice that no other segment of the university community has allowed. A medical research paper containing a subtle endorsement for a medication carries more weight with clinicians and patients if the pharmaceutical company that wrote the paper is not

This article was accepted for publication on May 3, 2011.

J. Leo $(\bowtie)$

DeBusk College of Osteopathic Medicine,

Lincoln Memorial University,

Harrogate, TN 37752, USA

e-mail: jonathan.leo@lmunet.edu

\section{J. R. Lacasse}

Center for Applied Behavioral Health Policy,

College of Public Programs, Arizona State University,

Phoenix, AZ 85004, USA

J. R. Lacasse

e-mail: jeffrey.lacasse@asu.edu

A. N. Cimino

College of Public Programs, Arizona State University,

Phoenix, AZ 85004, USA

A. N. Cimino

e-mail: andreacimino@mac.com mentioned in the authorship byline, especially if it lists prominent university professors from prestigious institutions.

The practice of ghostwriting is neither rare nor harmless. Alleged ghost authors haunt the clinical trial literature of virtually all the recent blockbuster drugs, including medicines like Vioxx, Avandia, Paxil, Zoloft, Zyprexa, hormonereplacement therapy, and Fen-phen. As the makers of these drugs are embroiled in product liability lawsuits from their product's tendency to cause harm, the involvement of ghostwriters in the production of scientific evidence to support their use has raised eyebrows.

Recently, a public dialogue on ghostwriting has emerged, with public advocacy organizations and some medical journal editors, practicing physicians and bioethicists voicing their perspectives and calling for reform. As this dialogue continues, we have noted a critically important issue often overlooked by many of those involved: When exactly should a scientific paper be considered ghostwritten? Medicine aside, most people who hear the term "ghostwriter" think of a paid writer who authors an article, book, or speech without credit. While discussions of ghostwriting have some complexities, it seems there is one, and only one, criterion to determine whether a scientific paper has been ghostwritten: If a person who should have been listed as an author was left off the byline, then the paper has been ghostwritten.

\section{Whiter Shades of Pale}

Instead, because of supposedly offsetting circumstances, various authors, companies, reporters, and university administrators have taken the stance that some scientific papers, which admittedly did involve a ghost author, should 
not be considered "ghostwritten." Defenses of ghostwritten papers are endlessly creative: The paper was peer reviewed. The named authors signed off on the content. The person who wrote most of the paper was acknowledged as an editorial assistant. All of the listed authors did a significant amount of work. The listed authors were involved in every step of the paper's development. The paper was published in a top-tier journal. The average reader cannot detect any commercial bias in the paper. The data were reported accurately. The college professors listed as authors were not paid. The listed author is a great scientist. The paper's conclusions are in-line with current thinking on the topic. However, if an author of a paper was not listed on the byline, then it is ghostwritten; no peripheral or subsequent events in the life of a scientific paper can erase its ghostwritten history. Further, there are no gradations of ghostwriting - either the paper involved a ghostwriter or it did not.

Rather than provide credible explanations why papers with unnamed authors are not ghostwritten, these justifications are best characterized as a set of conditions under which some academicians believe ghostwriting is permissible. This difference is critical, and shifts the argument in an important way: According to some in authority, inaccurate authorship bylines are acceptable if certain conditions are met.

A recent case of alleged ghostwriting involves the textbook "Recognition and Treatment of Psychiatric Disorders," published by the American Psychiatric Press (APP) in 1998. The named authors are Charles Nemeroff, current chairman of Psychiatry at the University of Miami, and Alan Schatzberg, former Chairman of Psychiatry at Stanford and former President of the American Psychiatric Association. Documents recently released as part of discovery in a law suit against GlaxoSmithKline have led to allegations that the textbook involved ghost authors employed by the manufacturer of Paxil. A seemingly damning draft of the book by the medical writers is available on the web, at http://pogoblog. typepad.com/pogo/gw-attachment-d.html. Complicating matters, the APP's trade journal, Psychiatric Times, defended the textbook's use of medical writers and declared that they stand behind the authorship line. At present, the validity of either the ghostwriting charges or the defenses are not conclusively known, but the problematic defense offered by APP gives a window into the wrong-headed way that the issue of ghostwriting is routinely perceived. APP believes that because Nemeroff and Schatzberg signed off on the final copy, the article was not ghostwritten. James Scully, Medical Director for the APA, even stated, "The book was reviewed for any potential bias (among other things) by eight independent reviewers, and there was no undue influence on the content from industry or any other outside source." Scully's statement show a certain disdain for readers and brings to mind the saying, "Trust us, we're experts." Instead of taking the word of an APA committee that the book is unbiased, shouldn't all the authors be listed so that readers can make up their own minds?

This case brings up several important points about ghostwriting. First, whether or not the named authors sign off on a final copy of a manuscript is immaterial in determining if it was ghostwritten. The pertinent question is, were there other authors deserving of credit that were not listed in the byline? Second, reviewers considered independent and unbiased by the APA, or another authoritative entity, determining a study received "no undue influence" from industry has absolutely nothing to do with determining ghost authorship.

Transparent and honest authorship bylines would seem to be a bare minimum standard for professors publishing medical research. As an analogy, consider the following scenario. A college professor determines one of his graduate students handed in a paper largely written by someone else. After being confronted, the student acknowledges that indeed someone else wrote the paper. The student then says, "I approved the final copy, and had eight classmates look over the paper and they all vouch for its unbiased conclusions". The professor correctly replies, "I don't think you understand the issues involved here." http:// pn.psychiatryonline.org/content/46/2/1.1.full

Of course, there is good reason to think "Recognition and Treatment of Psychiatric Disorders" contains significant bias. The makers of Paxil paid the alleged ghostwriter's employing firm $\$ 120,000$ to author the book. Daniel Carlat, a psychiatrist who has written for The New York Times, called the book, “... an advertisement for Paxil. ... analysis of the book's content shows that it was crafted to encourage primary care doctors to prescribe Paxil preferentially over its competitors, such as Zoloft." The used copy we ordered even had a sticker, "Compliments of Paxil" on both the front and rear cover. Clearly, physicians who received this book had reason to suspect that it was biased; shouldn't they also have had the benefit of an accurate authorship byline? See: http://carlatpsychiatry.blogspot.com/2010/12/ nemeroff-and-schatzbergs-textbook.html.

\section{Acknowledging Authors Hides Conflict-of-Interests}

Perhaps the most pernicious practice in ghostwriting involves thanking writers for providing "editorial assistance" in the acknowledgments section of the paper instead of the authorship byline, which essentially changes the rule of authorship attribution so that ghostwriting is acceptable. Several groups in medicine including the European Medical Writers Association (EMWA) sanction this practice. While the average reader likely interprets "editorial assistance" as 
help with grammar or improvements to the overall readability of the article, in reality, such "assistants" make major contributions to papers, and would commonsensically be considered co-authors. Tellingly, many medical writers are "editorial assistants" on some scientific papers, but co-authors on others. It would seem obvious that someone employed as a "medical writer" would be an author, but current dialogue on ghostwriting ignores such common-sense interpretations.

Listing ghost authors as editorial assistants allows pharmaceutical companies to publish articles with conflicts-ofinterest that are not transparently reported. Editorial assistants are not mentioned in the abstract, are not indexed in publication databases, are not mentioned in subsequent citations, and are never mentioned in news media accounts of the article. In other words, the fact that a pharmaceutical company directly co-authored the paper is concealed from view. That this is seen as acceptable in an era of increased disclosure of conflicts-of-interest is puzzling.

The problem with simply thanking ghostwriters in the acknowledgements section is clearly illustrated by Study 329, probably the most notorious ghostwritten paper in the medical literature. The study examined the use of Paxil in adolescents and concluded, "Paroxetine is generally well tolerated and effective for major depression in adolescents." Several years after the paper was published, court proceedings revealed internal company documents admitting that the study found that Paxil was not any better than placebo on the pre-registered outcome measures, and that the company was concerned about how to manage the negative findings. In reference to the clinical trial literature on the use of antidepressants in children, David Healy, a psychiatrist and medical historian, has referred to the difference between the actual data and the published papers as the greatest divide in all of medicine, and according to Healy most of it has been ghostwritten.

A fascinating series of documents, all available on the web, reveal the steps involved in Study 329's transformation from initial idea to final draft. Sally Laden, an employee of Scientific Therapeutics was hired by GlaxoSmithKline, the manufacturer of Paxil, and wrote the first draft. After each draft was submitted, she incorporated suggestions from some of the listed authors into each subsequent draft. But, rather than be listed as one of the 22 academic co-authors listed on the byline, Laden was only acknowledged for editorial assistance. According to the European Writers Association, since Laden was acknowledged, Study 329 was not ghostwritten, even though a major author remains invisible as far as the authorship byline - to the rest of us, the very definition of ghostwriting. If the medical community accepts the idea that mentioning medical writers in the acknowledgement section is acceptable, then they are effectively declaring that they consider ghostwriting a legitimate practice.

\section{Honorary Authorship is Distinct from Ghostwriting}

Other confusions exist between ghostwriting and honorary authorship. Honorary authorship involves an undeserving person getting author credit, whereas ghostwriting involves a deserving person not getting credit. The two do not necessarily go together. It is possible for the contributions of named authors on a paper to warrant authorship, but if someone else deserving of authorship credit did not appear in the byline, the paper has still been ghostwritten. The extent of the named authors' involvement in the paper is immaterial in determining whether the paper was ghostwritten; the extent of involvement of unnamed authors is of key importance.

Honorary authorship is relatively benign and poses little problem to readers. For example, if a reader of a paper subsequently learned that the author byline included an "undeserving" author, such as a department chair or a laboratory technician who collected data but did little meaningful work on the paper, this would probably not alter the reader's conclusions about the paper. A laboratory technician is unlikely to have an important bias or major influence over the interpretation of the data. Ghostwriting, on the other hand, is performed by writers who have undisclosed conflictsof-interests and are paid well by pharmaceutical companies to ensure that the manuscripts contain the chosen marketing messages. This wreaks havoc on the medical literature and may even negatively impact public health by distorting the efficacy of medications, an important issue in an era of evidence-based medicine.

\section{Context is Irrelevant}

Another common defense of ghostwriting revolves around the timing of the publication. Some may argue that if a paper with unnamed authors was published at a time when formal policies were not in place, then the paper should not be considered ghostwritten. Certainly, ghostwriting policies are evolving, but, logically, how authorities choose to handle known incidents of ghostwriting is a distinct issue from the question of whether a paper is ghostwritten.

There is also confusion about the role of payment in ghostwriting. There have been reports of academics allowing their names to be used on the byline of academic papers that they did not write in return for payment. A recent letter from NIH focused on this aspect of ghostwriting. While being paid for authorship is ethically problematic, it distracts from the core issue of ghostwriting - whether or not money changed hands is irrelevant to the question at hand. 


\section{Using Common Sense}

Some have argued that it is very difficult to discern who actually deserves to be placed on the byline of a research paper, and that we risk oversimplifying a complex issue. While there are some complexities, we worry that this defense could be used to maintain the status quo. We agree that there are individuals who contribute to papers but should not be on the authorship byline (e.g., a copyeditor, or a clinician who only treated patients but did not write anything). However, use of common sense may be productive in clarifying this issue. If a medical writer worked on a paper, well, writers are generally authors and should be listed on the byline. It may say something about the state of academic medicine that this proposition is seen as controversial. More specific criteria are possible. For instance, if an individual writes a first draft, or was paid to write substantive content beyond copyediting, that person should be listed as an author. The number of authors on medical research papers would increase by these methods, but it is difficult to see why this is a problem, or how this would be negative for practicing physicians, patients, or the scientific community. The well-accepted standard for managing conflicts-of-interest in academic medicine is full disclosure; increasing transparency by putting all medical writers on the byline hurts no one (except perhaps the pharmaceutical companies).

\section{Towards More Transparent Standards}

The International Committee of Medical Journal Examiners (ICMJE), a group of medical editors who have developed policies related to the medical publishing process, has proposed three criteria for determining who should be given a byline as author on scientific papers. These criteria are "1) substantive contributions to conception and design, acquisition of data, or analysis and interpretation of data; 2) drafting the article or revising it critically for important intellectual content; and 3) final approval of the version to be published." While these are now the traditional, oft-cited criteria for authorship, they do not address the contemporary concern of ghostwriting. In fact, although this is unintended, use of the ICMJE criteria may facilitate ghostwriting while creating the impression that medical journals have strict policies on authorship.

Consider this hypothetical situation: An industry-funded medical writer authors a paper in conjunction with academic researchers. The medical writer authors the 1st draft of the paper and makes many substantive edits, eventually writing $99 \%$ of the paper. Before the absolute "final" version is reached, the medical writer turns it over to the academic researchers, and never approves the final version; the medical writer is acknowledged for editorial assistance. Thus, an inaccurate byline and a ghost author are created, but the authors followed the ICMJE rules to the letter - $\mathrm{a}$ writer who does not approve the final manuscript cannot be an author. If accused of ghostwriting, all concerned can simply declare that they followed ICJME guidelines.

As ghost authoring gains more notice, some journals have adopted policies stricter than the ICMJE guidelines. For instance, the journal Neurology has instituted a much more stringent policy. Rather than asking who is an author per ICMJE criteria, they ask, "Who influenced the content?" and require that any paid medical writer be included in the author byline, accompanied by full disclosure. In their authorship standards, they define a ghostwriter as "an undisclosed person (paid or unpaid) who has made an intellectual contribution in writing the submitted manuscript." Basically, Neurology has formalized, for the medical literature, a pragmatic and intellectually sound definition of ghostwriting. Although results have yet to be evaluated, Neurology's policy is a very positive step in the right direction, and something that should be adopted more widely.

\section{Where Do We Go from Here?}

Due to its secretive nature, it is difficult to quantify how many invisible corporate authors haunt the medical literature. Collaboration has always been important for scientific progress, and many clinical trials and review papers are joint projects between pharmaceutical company employees and university professors. Medical writers are an important component of academic-medical publishing, and there is no reason to end industry-academic cooperation, rather such collaboration should be transparent. As long as the papers resulting from these projects accurately and transparently report all authors in the author byline, there seems to be little need for concern. Listing all the authors involved with a paper in the byline is not complicated and in principal resolves the issue of ghostwriting.

To improve the credibility of biomedical research eroded by ghostwriting, the ICMJE should revise their approach to regulating authorship. As authors submit manuscripts to medical journals, each author should be required to sign a statement guaranteeing that no ghost authors participated in authorship or co-authorship of the submitted article, and that all medical writers are listed as authors on the byline. This simple move forward could have profound consequences for the medical literature as a whole. Much progress has been made relatively quickly in identifying ghostwriting as a problem, and we are now on the verge of largely solving this problem-if those with editorial influence in academic medicine will insist on policies to 
ensure that there are no undisclosed conflicts of interest haunting the contemporary medical literature.

\section{Further Reading}

Brian, D., \& Thacker, P. 2010. POGO letter to NIH on ghostwriting academics [Internet]. Washington, DC; Nov 29 [cited 2011 Feb 2]. Project on Government Oversight. Available from: http://www. pogo.org/pogo-files/letters/public-health/ph-iis-20101129.html.

Collins, F. S. 2010. NIH response letter to Paul Thacker POGO on ghostwriting academics [Internet]. Washington, DC; Feb 17 [cited 2011 Feb 2]. Project on Government Oversight. Available from: http://pogoblog.typepad.com/pogo/nih-response-to-pogoon-ghostwriting-20110217.html.

Healy, D., \& Cattell, D. 2003. Interface between authorship, industry and science in the domain of therapeutics. British Journal of Psychiatry [Internet]. Jul 1 [cited 2011 Feb 2];183(1):22-27. Available from: http://bjp.rcpsych.org/cgi/content/abstract/183/1/22.

Jureidini, J. N., McHenry, L. B., \& Mansfield, P. R. 2008. Clinical trials and drug promotion: Selective reporting of study 329. International Journal of Risk and Safety in Medicine, 20(1-2), 73-81.

Lacasse, J. R., \& Leo, J. 2010. Ghostwriting at elite academic medical centers in the United States. PLoS Medicine [Internet]. Feb 2;7 (2):e1000230. Available from: http://www.plosmedicine.org/article/ info\%3Adoi\%2F10.1371\%2Fjournal.pmed.10002 International Committee of Medical Journal Editors.
Leo, J., \& Lacasse, J.R. 2010. Ghostwriting and Academic Medicine. Chronicle of higher education. [Internet] Jul 19, 2010. Available at: http://chronicle.com/article/GhostwritingAcademic/123613/

Moffatt, B., \& Elliott, C. 2007. Ghost marketing: pharmaceutical companies and ghostwritten journal articles. Perspective Biology and Medicine [Internet]. [cited $2011 \mathrm{Feb} 2$ ];50(1):18-31. Available from: http://www.webcitation.org/5wJIVyU8j.

Sismondo, S. 2007. Ghost management: how much of the medical literature is shaped behind the scenes by the pharmaceutical industry? PLoS Medicine [Internet]. Sep [cited 2011 Feb 2];4 (9):e286. Available from: http://dx.doi.org/10.1371/journal. pmed.0040286.

2009. Uniform requirements for manuscripts submitted to biomedical journals: Ethical considerations in the conduct and reporting of research: Authorship and contributorship [Internet]. Available from: http://www.icmje.org/ethical_1author.html.

Jonathan Leo is Professor of Neuroanatomy at Lincoln Memorial University in Harrogate TN.

Jeffrey Lacasse is Assistant Professor of Social Work at Arizona State University. Leo and Lacasse have published several articles on medical ghostwriting and the chemical imbalance theory of depression.

Andrea Cimino is a doctoral student at Arizona State University. 